\title{
Identification of SLED1 as a Potential Predictive Biomarker and Therapeutic Target of Post-Infarct Heart Failure by Bioinformatics Analyses
}

\author{
Jiajia Zhang, ${ }^{1} \mathrm{MB}$, Jun Wang, ${ }^{1} \mathrm{PhD}$, Yong Wu, ${ }^{1} \mathrm{MD}$, Wei Li, ${ }^{1} \mathrm{MS}$, \\ Kaizheng Gong, ${ }^{1} \mathrm{PhD}$ and Pei Zhao, ${ }^{1} \mathrm{MD}$
}

\begin{abstract}
Summary
The aim of this study was to explore potential predictive biomarkers and therapeutic targets of post-infarct heart failure (HF) using bioinformatics analyses.

CEL raw data of GSE59867 and GSE62646 were downloaded from the GEO database. Differentially expressed genes (DEGs) between patients with ST-segment elevation myocardial infarction (STEMI) and those with stable coronary artery disease (CAD) at admission and DEGs between admission and 6 months after myocardial infarction (MI) in patients with STEMI were analyzed. A gene ontology (GO) analysis and a gene set enrichment analysis (GSEA) were performed, and a protein-protein interaction network was constructed. Critical genes were further analyzed.

In total, 147 DEGs were screened between STEMI and CAD at admission, and 62 DEGs were identified in patients with STEMI between admission and 6 months after MI. The results of GO and GSEA indicate that neutrophils, neutrophil-related immunity responses, and monocytes/macrophages play important roles in MI pathogenesis. SLED1 expression was higher in patients with HF than in those without HF at admission and 1 month after MI. GSEA indicates that mTORC1 activation, E2F targets, G2M checkpoint, and MYC targets v1 inhibition may play key roles in the development of post-infarct HF. Furthermore, SLED1 may be involved in the development of post-infarct HF by activating mTORC1 and inhibiting E2F targets, G2M checkpoint, and MYC targets v1.
\end{abstract} disease.

SLED1 may be a novel biomarker of post-infarct HF and may serve as a potential therapeutic target in this

Key words: Acute myocardial infarction, Gene set enrichment analysis

(Int Heart J 2021; 62: 23-32)

$\mathrm{C}$ ardiovascular diseases are the leading causes of death worldwide, including in low-income and middle-income countries. ${ }^{1}$ In 2013, the total number of deaths resulting from cardiovascular diseases reached almost $33 \%$ of all deaths globally. ${ }^{2}$ Among these, ischemic heart disease accounts for $47 \% .^{2}$

Owing to the implementation of primary percutaneous coronary intervention and the prescription of evidence-based drug treatments, the prognosis of STsegment elevation myocardial infarction (STEMI) has improved markedly over the past 20 years. ${ }^{3)}$ Despite these advances, STEMI leads to irreversible loss of myocardial cells and adverse left ventricular remodeling. Approximately $50 \%$ of the cases demonstrate a lower ejection fraction after myocardial infarction (MI). ${ }^{3)}$ Moreover, 20\% of patients with anterior STEMI may suffer from heart failure (HF) within 1 year. ${ }^{4)}$ The long-term prognosis of patients with STEMI remains poor. Therefore, an in-depth understanding of the pathophysiology of STEMI and post- infarct HF is still required to identify new prevention and treatment targets. In addition, effective and predictive biomarkers for post-infarct HF are urgently required for risk stratification, prognosis assessment, and individualized medicine.

The present study aimed to search for differentially expressed genes (DEGs) in patients with STEMI at admission that may be involved in the pathogenesis of STEMI and then evaluate these genes to identify reliable predictive biomarkers and therapeutic targets for post-infarct HF.

\section{Methods}

Microarray data information: CEL raw data files of GSE59867 $7^{5}$ and GSE62646 ${ }^{6}$ were downloaded from the GEO database, ${ }^{7,8}$ and they were all based on the GPL6244 platform of [HuGene-1_0-st] Affymetrix Human Gene 1.0 ST Array [transcript (gene) version]. The GSE59867 dataset contained 436 peripheral blood mononuclear cell

From the ${ }^{1}$ Department of Cardiology, The Affiliated Hospital of Yangzhou University, Yangzhou University, Yangzhou, China.

This work was supported by National Natural Science Foundation of China (NSFC-81970225).

Address for correspondence: Pei Zhao, MD, Department of Cardiology, The Affiliated Hospital of Yangzhou University, Yangzhou University, No. 368 Hanjiang Middle Road, Yangzhou 225001, China. E-mail: zhaopei@yzu.edu.cn

Received for publication June 18, 2020. Revised and accepted September 25, 2020.

doi: 10.1536/ihj.20-439

All rights reserved by the International Heart Journal Association. 


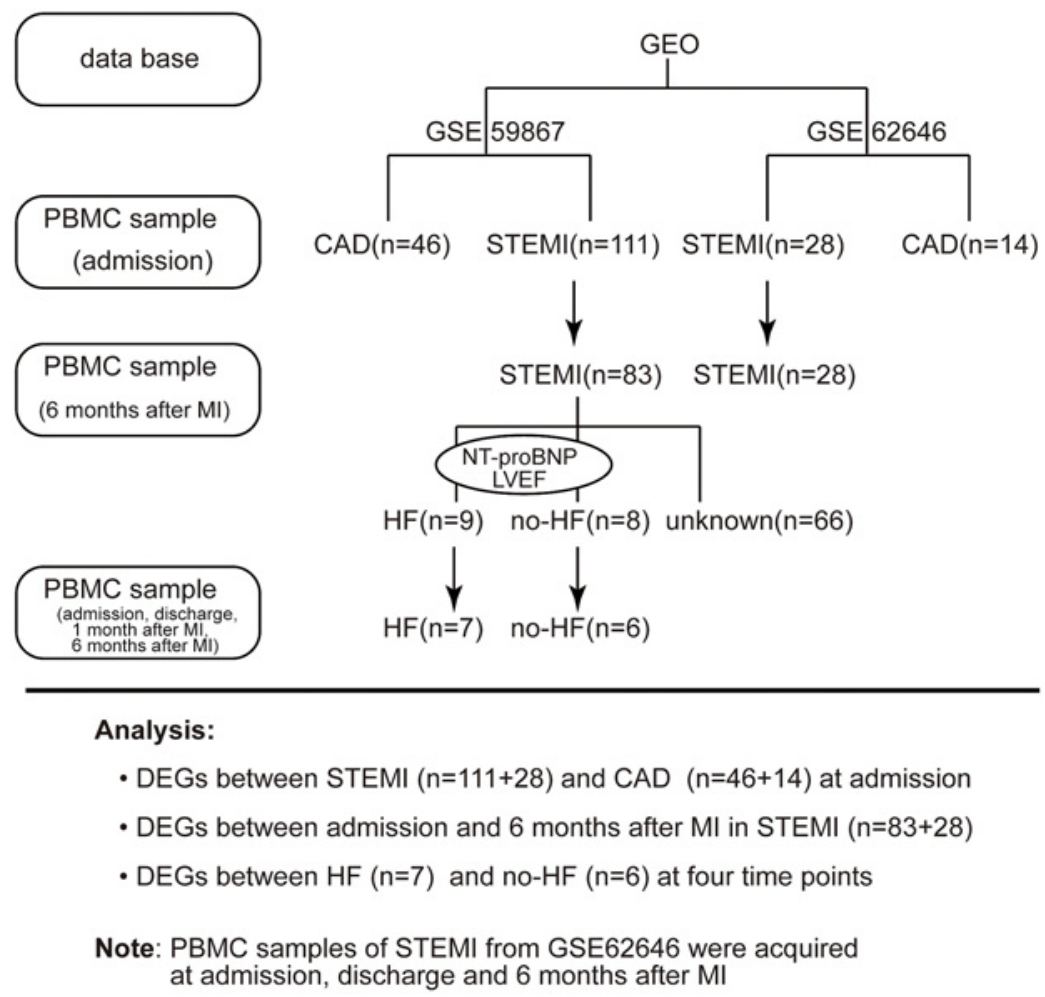

Figure 1. Flow chart of the selection process.

(PBMC) samples from 111 patients with STEMI at four time points (admission, discharge, 1 month after MI, and 6 months after MI) and from 46 patients with stable coronary artery disease (CAD) at admission as the control. Among the patients with STEMI, nine were diagnosed with HF, and eight were excluded from HF. The GSE 62646 dataset included 98 PBMC samples from 28 patients with STEMI at three time points (admission, discharge, and 6 months after MI) and from 14 patients with $\mathrm{CAD}$ at admission as the control. From the two datasets, all patients with STEMI underwent coronary angiography and angioplasty of the infarct-related artery, and all patients with CAD were confirmed using coronary angiography (at least one stenosis exceeding $50 \%$ ) or by previous coronary angioplasty, coronary artery bypass graft, or noninvasive tests (positive exercise test).

In this study, PBMC gene expression data from 139 patients with STEMI (111 from GSE59867 and 28 from GSE62646) at admission were compared with those from 60 patients with CAD (46 from GSE59867 and 14 from GSE62646) to identify DEGs in patients with STEMI at admission for subsequent analysis. Among the 139 patients with STEMI, 111 patients (83 from GSE59867 and 28 from GSE62646) had gene expression data at both admission and 6 months after STEMI. The paired data of the 111 patients were analyzed to screen for DEGs in patients with STEMI at admission for subsequent analysis. In total, complete gene expression data at four time points (admission, discharge, 1 month after MI, and 6 months after MI) were available from seven patients with $\mathrm{HF}$ and six patients without HF. These data were used to identify biomarkers for post-infarct HF for further analysis. The clinical characteristics and medicines of patients for postinfarct HF analysis were analyzed in the original author's paper. ${ }^{5)}$ There was no significant difference between patients with and without HF, except for left ventricular ejection fraction, NT proBNP, and diuretics. (Figure 1)

Microarray data preprocessing: CEL raw data of GSE 59867 and GSE62646 were merged and preprocessed using the affy package ${ }^{9)}$ in $\mathrm{R}$ Bioconductor for background correction, normalization, and calculation expression. The $\mathrm{Sva}^{10)}$ and limma ${ }^{11,12)}$ packages in $\mathrm{R}$ Bioconductor were used for removal of batch effects and for normalization between arrays, respectively. The probes were converted into gene symbols according to the probe annotation information. Probes without the corresponding gene symbols were removed. If multiple probes corresponded to the same gene symbol, the probe with the highest expression value was retained.

DEGs analysis: DEGs between patients with STEMI and patients with $\mathrm{CAD}$ at admission were identified using the limma package. The paired $t$-test of the limma package was used to identify DEGs between admission and 6 months after MI in patients with STEMI. A fold change of a gene expression ratio $>1.0$ and adjusted $P$-value (adj. $P)<0.05$ were used as cut-off criteria. Genes with a fold change of gene expression ratio $>2.0$ were chosen as top DEGs. DEGs between HF and non-HF at the four time points were also identified using the limma package. A fold change of a gene expression ratio $>1.0$ and $P<0.05$ were used as the threshold value. Subsequently, DEGs of patients with STEMI at admission were visualized as vol- 
A

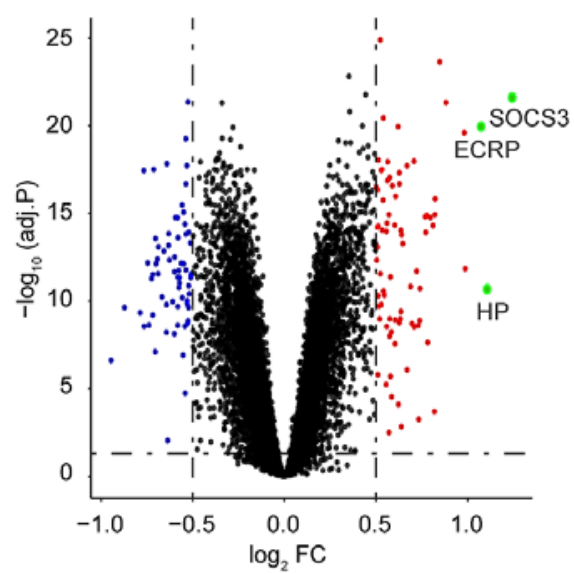

C

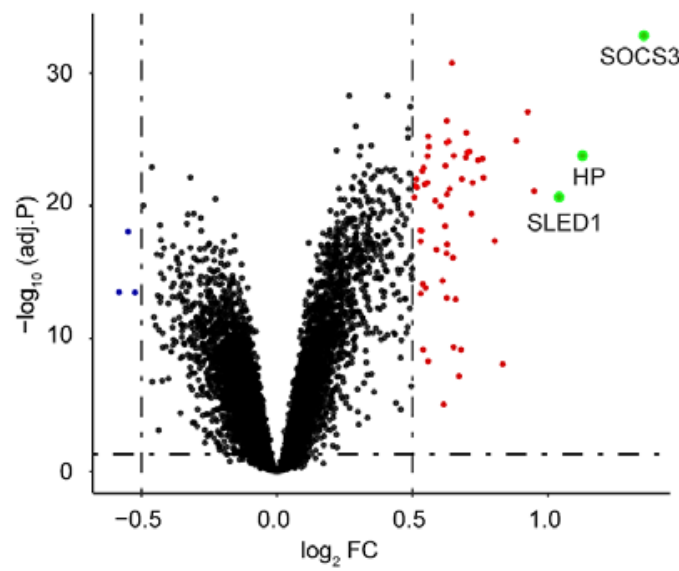

B CON-admission

STEMI-admission

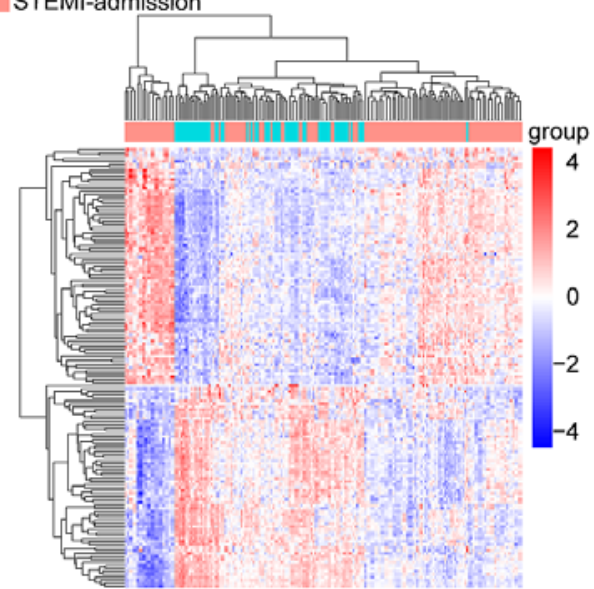

D STEMI-6M

STEMI-admission

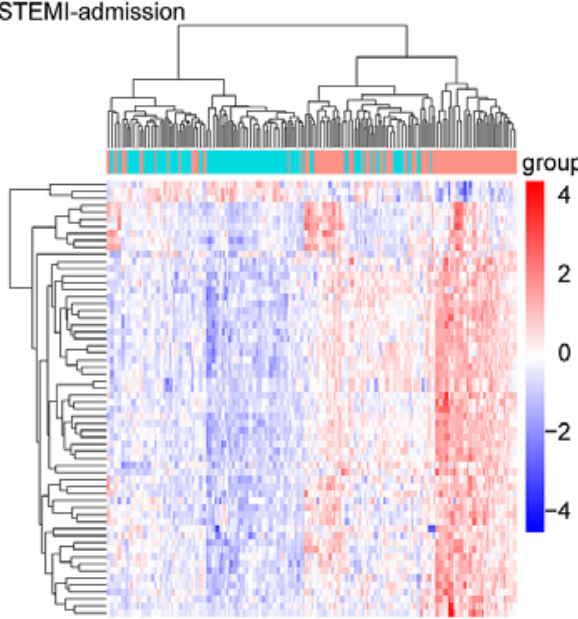

Figure 2. Identification of DEGs. A: Volcano plot of mRNA expression profiles discriminating STEMI from CAD. B: Heatmap from cluster analysis of 147 DEGs between STEMI and CAD at admission. C: Volcano plot of mRNA expression profiles between admission and 6 months after MI in patients with STEMI. D: Heatmap from cluster analysis of 62 DEGs between admission and 6 months after MI in patients with STEMI. Red and blue dots in volcano plots represent upregulated and downregulated mRNAs, respectively. Green dots are the top DEGs. Each column in heatmaps represents a sample, and each row represents a gene. The gradual color change from blue to red indicates the changing process from downregulation to upregulation in heatmaps. A fold change of gene expression ratio $>1.0$ and adj. $P<0.05$ were considered statistically significant.

cano plots and heatmaps using the ggplot $2^{13)}$ and pheatmap ( https://cran.r-project.org/web/packages/pheatmap) packages, respectively, in $\mathrm{R}$.

Gene ontology (GO) functional enrichment analysis of DEGs: To explore the potential function of DEGs in patients with STEMI, DEGs were subjected to GO enrichment analysis to determine the associated biological process (BP), molecular function (MF) and cellular component (CC) terms using the clusterProfiler package ${ }^{14)}$ in R Bioconductor. Adj. $P<0.05$ and enrichment count $\geq 2$ were used as the threshold values.

Gene set enrichment analysis (GSEA): GSEA ${ }^{15)}$ is used to determine whether a predefined gene set is significantly different between two groups. In this study, GSEA was performed using hallmark gene sets (http://software.broadi nstitute.org/gsea/msigdb/collections.jsp\#H). To explore the mechanism of MI and post-infarct HF, GSEA was con- ducted using the clusterProfiler package in $\mathrm{R}$ Bioconductor. The entire gene lists were ranked according to the log 2 fold change of gene expression ratios between STEMI and $\mathrm{CAD}$ at admission and between admission and 6 months after MI in patients with STEMI, respectively. Adj. $P<0.05$ was considered statistically significant.

Protein-protein interaction (PPI) networks of DEGs: To screen for crucial genes associated with STEMI, DEGs were mapped into the Search Tool for the Retrieval of Interacting Genes database (STRING; Version 11.0) ${ }^{16)}$ to predict PPI pairs with a combined score $>0.4$. The PPI networks were visualized using Cytoscape software (Version 3.6.1). ${ }^{17}$ Nodes with a degree of interaction $\geq 10$ were considered as hub genes. Additionally, the Molecular Complex Detection plugin (MCODE, Version 1.5.1, http:// apps.cytoscape.org/apps/mcode) in Cytoscape was applied to identify a function module in the PPI network with the 
Table I. The Overlapping GO Terms Enriched by DEGs in Patients with STEMI

\begin{tabular}{|c|c|c|c|c|c|c|}
\hline \multirow{2}{*}{ GO } & \multirow{2}{*}{ ID } & \multirow{2}{*}{ Description } & \multicolumn{2}{|c|}{ DEGs (MI-CAD) } & \multicolumn{2}{|c|}{ DEGs (MI-Follow) } \\
\hline & & & Count & adj. $P$ & Count & adj. $P$ \\
\hline BP & GO: 0042119 & neutrophil activation & 17 & 0.00028598 & 12 & 0.0000245 \\
\hline BP & GO: 0002283 & neutrophil activation involved in immune response & 16 & 0.0002963 & 11 & 0.0000796 \\
\hline BP & GO: 0032103 & positive regulation of response to external stimulus & 13 & 0.0002963 & 10 & 0.0000245 \\
\hline BP & GO: 0043312 & neutrophil degranulation & 16 & 0.0002963 & 11 & 0.0000796 \\
\hline BP & GO: 0002446 & neutrophil mediated immunity & 16 & 0.00031838 & 11 & 0.0000828 \\
\hline BP & GO: 0002683 & negative regulation of the immune system process & 14 & 0.00139748 & 6 & 0.02523409 \\
\hline BP & GO: 1902106 & negative regulation of leukocyte differentiation & 7 & 0.0024105 & 3 & 0.03087566 \\
\hline BP & GO: 0060326 & cell chemotaxis & 11 & 0.00300653 & 8 & 0.00046973 \\
\hline BP & GO: 0050727 & regulation of the inflammatory response & 13 & 0.00316498 & 11 & 0.0000329 \\
\hline BP & GO: 0097529 & myeloid leukocyte migration & 9 & 0.00316498 & 7 & 0.00045122 \\
\hline BP & GO: 0071677 & positive regulation of mononuclear cell migration & 4 & 0.00341911 & 2 & 0.02523409 \\
\hline BP & GO: 0030595 & leukocyte chemotaxis & 9 & 0.00531431 & 7 & 0.00053106 \\
\hline BP & GO: 0071674 & mononuclear cell migration & 6 & 0.00595344 & 4 & 0.00436075 \\
\hline BP & GO: 1903706 & regulation of hemopoiesis & 12 & 0.00785638 & 6 & 0.02227269 \\
\hline BP & GO: 0045088 & regulation of the innate immune response & 11 & 0.00986956 & 6 & 0.01497313 \\
\hline $\mathrm{BP}$ & GO: 0060670 & branching involved in the labyrinthine layer morphogenesis & 3 & 0.00986956 & 3 & 0.000604 \\
\hline BP & GO: 0002548 & monocyte chemotaxis & 5 & 0.01180963 & 3 & 0.01633613 \\
\hline BP & GO: 0002688 & regulation of leukocyte chemotaxis & 6 & 0.01480557 & 4 & 0.00943495 \\
\hline BP & GO: 0014912 & negative regulation of smooth muscle cell migration & 4 & 0.01480557 & 2 & 0.04260046 \\
\hline BP & GO: 1902105 & regulation of leukocyte differentiation & 9 & 0.01480557 & 6 & 0.00491112 \\
\hline BP & GO: 0071675 & regulation of mononuclear cell migration & 4 & 0.02338211 & 3 & 0.00825707 \\
\hline BP & GO: 0002687 & positive regulation of leukocyte migration & 6 & 0.02368844 & 4 & 0.01199788 \\
\hline BP & GO: 0031349 & positive regulation of the defense response & 11 & 0.02462593 & 7 & 0.00840837 \\
\hline $\mathrm{BP}$ & GO: 0090026 & positive regulation of monocyte chemotaxis & 3 & 0.02462593 & 2 & 0.01985968 \\
\hline BP & GO: 0002685 & regulation of leukocyte migration & 7 & 0.02723273 & 4 & 0.02617203 \\
\hline BP & GO: 0002690 & positive regulation of leukocyte chemotaxis & 5 & 0.02723273 & 4 & 0.00431101 \\
\hline BP & GO: 0050921 & positive regulation of chemotaxis & 6 & 0.02723273 & 4 & 0.01361885 \\
\hline BP & GO: 0060713 & labyrinthine layer morphogenesis & 3 & 0.03156755 & 3 & 0.00165716 \\
\hline BP & GO: 0002573 & myeloid leukocyte differentiation & 7 & 0.03740316 & 4 & 0.02971064 \\
\hline BP & GO: 0032102 & negative regulation of response to external stimulus & 9 & 0.03965365 & 5 & 0.03087566 \\
\hline $\mathrm{BP}$ & GO: 0090025 & regulation of monocyte chemotaxis & 3 & 0.03965365 & 2 & 0.02763815 \\
\hline BP & GO: 0001960 & negative regulation of the cytokine-mediated signaling pathway & 4 & 0.0397247 & 3 & 0.01361885 \\
\hline BP & GO: 0042063 & gliogenesis & 8 & 0.0397247 & 5 & 0.01907026 \\
\hline BP & GO: 0050900 & leukocyte migration & 11 & 0.0397247 & 8 & 0.00342591 \\
\hline BP & GO: 0006700 & C21-steroid hormone biosynthetic process & 3 & 0.03984278 & 2 & 0.02824021 \\
\hline BP & GO: 0030593 & neutrophil chemotaxis & 5 & 0.04116033 & 5 & 0.00100868 \\
\hline BP & GO: 0050920 & regulation of chemotaxis & 7 & 0.04116033 & 4 & 0.03377804 \\
\hline $\mathrm{BP}$ & GO: 0060669 & embryonic placenta morphogenesis & 3 & 0.04116033 & 3 & 0.00268583 \\
\hline BP & GO: 0001959 & regulation of the cytokine-mediated signaling pathway & 6 & 0.04251596 & 4 & 0.01907026 \\
\hline BP & GO: 0060761 & negative regulation of response to cytokine stimulus & 4 & 0.04251596 & 3 & 0.01497313 \\
\hline BP & GO: 0001819 & positive regulation of cytokine production & 10 & 0.04430991 & 7 & 0.00817951 \\
\hline BP & GO: 1901342 & regulation of vasculature development & 10 & 0.04430991 & 9 & 0.00053106 \\
\hline $\mathrm{BP}$ & GO: 0045444 & fat cell differentiation & 7 & 0.04439439 & 6 & 0.00222828 \\
\hline $\mathrm{CC}$ & GO: 0070820 & tertiary granule & 8 & 0.00306951 & 6 & 0.0009739 \\
\hline $\mathrm{CC}$ & GO: 0042581 & specific granule & 7 & 0.01032844 & 5 & 0.0056212 \\
\hline $\mathrm{CC}$ & GO: 0030667 & secretory granule membrane & 9 & 0.01174884 & 5 & 0.01970976 \\
\hline $\mathrm{CC}$ & GO: 0035579 & specific granule membrane & 5 & 0.01766586 & 4 & 0.0056212 \\
\hline $\mathrm{CC}$ & GO: 0101003 & ficolin-1-rich granule membrane & 4 & 0.02434274 & 3 & 0.01242826 \\
\hline MF & GO: 0038024 & cargo receptor activity & 5 & 0.029224 & 4 & 0.02420311 \\
\hline MF & GO: 0004896 & cytokine receptor activity & 5 & 0.02984443 & 3 & 0.03924022 \\
\hline
\end{tabular}

DEGs (MI-CAD) indicates DEGs between STEMI and coronary artery disease at admission; DEGs (MI-Follow), DEGs between admission and 6 months after myocardial infarction in patients with STEMI; BP, biological process; CC, cellular component; DEGs, differentially expressed genes; GO, gene ontology; MF, molecular function; and STEMI, ST-segment elevation myocardial infarction.

following parameters: degree cut-off: 2 , node score cutoff: 0.2 , k-core: 2, maximum depth: 100. Modules with MCODE scores $\geq 4.5$ and nodes $\geq 5$ were used for further analysis.

Biomarker screening for post-infarct $\mathrm{HF}$ and function analysis: To seek biomarkers of post-infarct HF, top
DEGs, hub genes, and genes in the selected function modules were further analyzed to elucidate their roles in post-infarct HF during follow-up. Biomarkers were screened by crossing these genes with DEGs between patients with $\mathrm{HF}$ and those without $\mathrm{HF}$ at four time points. The biomarkers were further subjected to GSEA to ex- 
A

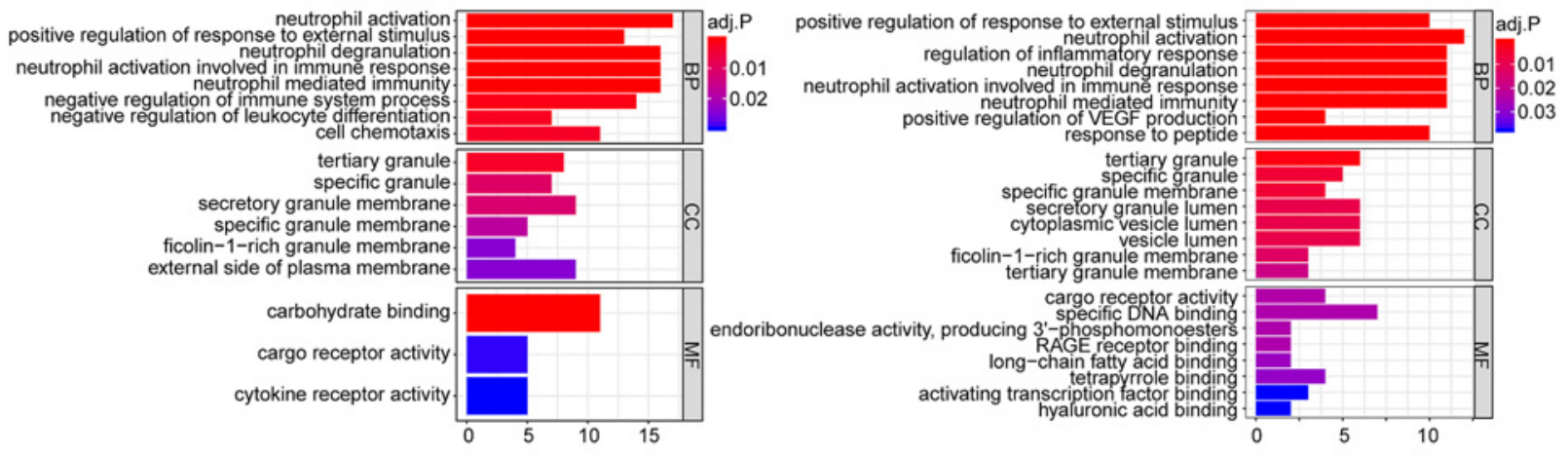

C

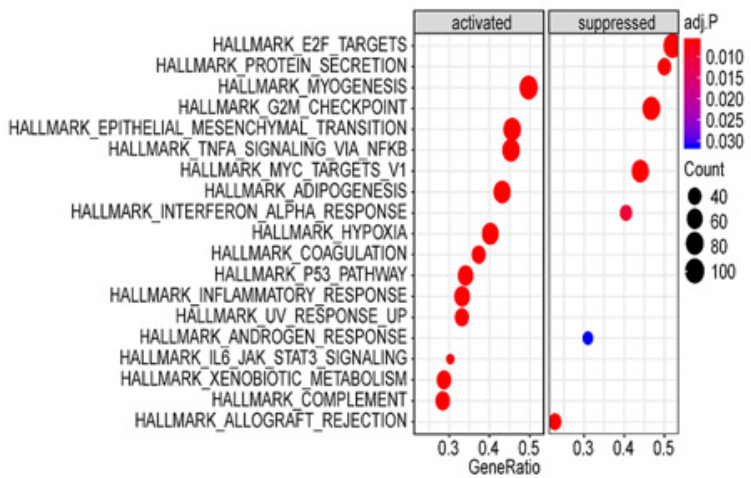

D

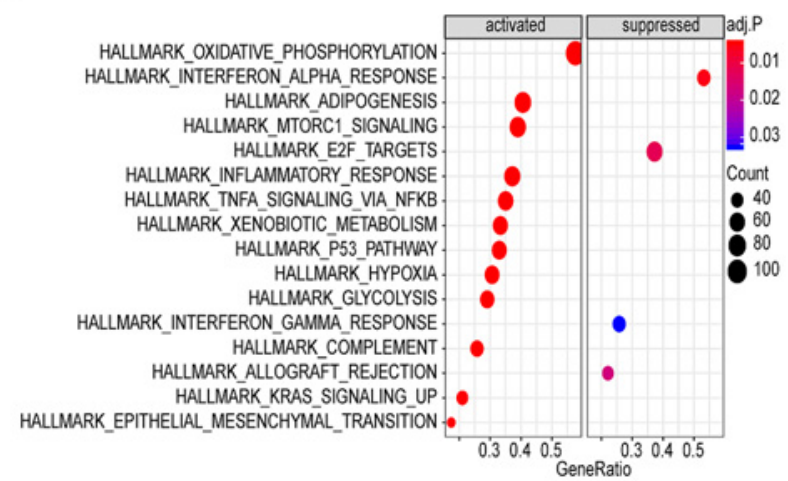

Figure 3. Mechanism analysis of STEMI. GO function enrichment analysis of DEGs between STEMI and CAD at admission (A) and between admission and 6 months after MI in patients with STEMI $(\mathbf{B})$. The change of color from red to blue indicates the adj. $P$ from low to high. The horizontal axes represent gene counts of enriched genes in corresponding GO terms. Adj. $P<0.05$ was considered statistically significant. GSEA of entire genes according to the $\log 2$ fold change of the gene expression ratio between STEMI and CAD at admission $(\mathbf{C})$ and between admission and 6 months after MI in patients with STEMI (D). The size and the color intensity of a circle represent the numbers of enriched genes and adj. $P$, respectively. Adj. $P<0.05$ was considered statistically significant.

plore their function in HF. The entire gene lists were ranked according to the correlation coefficient of a biomarker and other genes of gene expression ratio between patients with and without $\mathrm{HF}$ at admission. Adj. $P<0.05$ was considered statistically significant.

\section{Results}

Identification of DEGs: Raw data comprised 32,321 probes. A total of 18822 genes remained following data preprocessing. Subsequently, 147 DEGs were screened between STEMI and CAD at admission, including 79 upregulated and 68 downregulated genes (Figure 2A and B). As shown in Figure 2A, SOCS3, ERCP, and HP were the top DEGs. There were 62 DEGs in patients with STEMI between admission and 6 months after MI. Interestingly, 95\% (59 out of 62) of the DEGs were upregulated. SOCS3, HP, and SLED1 were identified as the top DEGs. The results are presented in volcano plots and heatmaps in Figure 2C and D.

Mechanism analysis of STEMI: $66 \mathrm{GO}$ terms were enriched in DEGs between STEMI and CAD, whereas 319 GO terms were enriched in DEGs between admission and 6 months after MI in patients with STEMI. There were 50 overlapping GO terms between the two sets of DEGs (Ta- ble I), which more likely indicate the functions of DEGs in STEMI. The top 8 enrichment terms are shown in graphs for each part of the GO analyses (if any) for the two sets of DEGs (Figure $3 \mathrm{~A}$ and $\mathrm{B}$ ). As illustrated in Figure $3 \mathrm{~A}$ and $\mathrm{B}, \mathrm{BP}$ enrichment analyses show that DEGs chiefly participated in neutrophil-related biological processes, such as neutrophil activation, neutrophil degranulation, and neutrophil-mediated immunity. In CC enrichment analysis, DEGs were predominantly involved in the construction of tertiary granule, specific granule, and secretory granule membrane. In MF terms, DEGs were mainly related to cargo receptor activity.

Twenty-seven hallmark gene sets were chosen for STEMI when compared with CAD, and 30 hallmark gene sets were screened for STEMI when compared with 6 months after MI. There were 21 overlapping hallmark gene sets enriched in STEMI (Table II), which may be related to the development of MI. As illustrated in Figure 3 $\mathrm{C}$ and $\mathrm{D}$, the overlapping activated hallmark gene sets mainly included TNF- $\alpha$ signaling via NF-kB, the inflammatory response, the P53 pathway, complement, and glycolysis. By contrast, E2F targets, the interferon alpha response, and allograft rejection were the overlapping suppressed hallmark gene sets.

PPI network construction and analysis: A PPI network 
Table II. The Overlapping Hallmark Gene Sets Enriched in Patients with STEMI

\begin{tabular}{lcccc}
\hline Description & \multicolumn{2}{c}{ MI-CAD } & \multicolumn{3}{c}{ MI-Follow } \\
& enrichmentScore & adj. $P$ & enrichmentScore & adj. $P$ \\
\hline HALLMARK_TNFA_SIGNALING_VIA_NFKB & 0.522071286 & 0.00628457 & 0.698989769 & 0.00448029 \\
HALLMARK_IL6_JAK_STAT3_SIGNALING & 0.49293494 & 0.00628457 & 0.593655818 & 0.00448029 \\
HALLMARK_INFLAMMATORY_RESPONSE & 0.482613528 & 0.00628457 & 0.595852774 & 0.00448029 \\
HALLMARK_REACTIVE_OXIGEN_SPECIES_PATHWAY & 0.477174119 & 0.01036055 & 0.685752564 & 0.00448029 \\
HALLMARK_P53_PATHWAY & 0.445908687 & 0.00628457 & 0.575456712 & 0.00448029 \\
HALLMARK_XENOBIOTIC_METABOLISM & 0.40940348 & 0.00628457 & 0.536417408 & 0.00448029 \\
HALLMARK_MYOGENESIS & 0.403026887 & 0.00628457 & 0.373873455 & 0.02232855 \\
HALLMARK_EPITHELIAL_MESENCHYMAL_TRANSITION & 0.393257704 & 0.00628457 & 0.478674726 & 0.00448029 \\
HALLMARK_HYPOXIA & 0.388079457 & 0.00628457 & 0.5407072 & 0.00448029 \\
HALLMARK_COAGULATION & 0.387177088 & 0.00628457 & 0.461673775 & 0.00448029 \\
HALLMARK_COMPLEMENT & 0.381198929 & 0.00628457 & 0.49184231 & 0.00448029 \\
HALLMARK_UV_RESPONSE_UP & 0.37520927 & 0.00628457 & 0.510961287 & 0.00448029 \\
HALLMARK_ADIPOGENESIS & 0.369588076 & 0.00628457 & 0.578860962 & 0.00448029 \\
HALLMARK_DNA_REPAIR & 0.334003156 & 0.02342579 & 0.415918467 & 0.02076412 \\
HALLMARK_GLYCOLYSIS & 0.329119185 & 0.01043841 & 0.486867778 & 0.00448029 \\
HALLMARK_ESTROGEN_RESPONSE_LATE & 0.320294174 & 0.01506932 & 0.430635724 & 0.00448029 \\
HALLMARK_APOPTOSIS & 0.309286511 & 0.03063726 & 0.438760087 & 0.00448029 \\
HALLMARK_KRAS_SIGNALING_UP & 0.291704593 & 0.03238866 & 0.422024238 & 0.00448029 \\
HALLMARK_ALLOGRAFT_REJECTION & -0.343867483 & 0.00628457 & -0.365514253 & 0.01707262 \\
HALLMARK_INTERFERON_ALPHA_RESPONSE & -0.378088157 & 0.01036055 & -0.575753057 & 0.00562873 \\
HALLMARK_E2F_TARGETS & -0.445985931 & 0.00628457 & -0.360133485 & 0.01218621 \\
\hline
\end{tabular}

MI-CAD indicates a gene set enrichment analysis between STEMI and coronary artery disease at admission; MI-Follow, a gene set enrichment analysis between admission and 6 months after myocardial infarction in patients with STEMI; and STEMI, ST-segment elevation myocardial infarction.

was constructed using STRING with DEGs between STEMI and CAD, including 72 (49 upregulated and 23 downregulated) nodes and 150 edges (Figure 4A). Furthermore, seven hub genes and two modules were screened from the PPI network (Figure 4B). Another PPI network was built with DEGs between admission and 6 months after MI in patients with STEMI, including 43 (41 upregulated and 2 downregulated) nodes and 105 edges (Figure 4C). In addition, five hub genes and one module were chosen from the PPI network (Figure 4D).

Mechanism analysis of post-infarct HF: GSEA indicated that 29 hallmark gene sets were enriched in patients with HF. The top 15 gene sets are depicted in Figure 5D. Compared with GSEA between STEMI and CAD, mTORC1 signaling activation was specific for post-infarct HF. In addition, E2F targets, G2M checkpoint, and MYC targets v1 were the suppressed hallmark gene sets.

Biomarkers of post-infarct $\mathrm{HF}$ and function analysis: A total of 29 genes, including top DEGs, hub genes, and genes in the selected function modules, were selected for further analysis to elucidate their role in post-infarct HF. At admission, the expression levels of CD163, FPR2, IL1 R2, SLED1, and VSIG4 were higher in patients with HF than in those without it (Figure 5A). Patients with $\mathrm{HF}$ showed higher expression of FOS and lower expression of KLRF1 than those in patients without HF at discharge (Figure 5B). The expressions of PTGS2 and SLED1 were upregulated in patients with HF 1 month after MI compared to those in patients without HF (Figure 5C). There was no expression difference among the 29 genes between patients with and without HF 6 months after MI. SLED1 was upregulated in patients with $\mathrm{HF}$ at admission and 1 month after MI when compared to that in patients without
HF. Therefore, SLED1 may be a potential biomarker of post-infarct HF.

GSEA of SLED1 was carried out between patients with and without $\mathrm{HF}$ at admission to explore the function of SLED1 in post-infarct HF, which showed that 32 hallmark gene sets were related to SLED1. Interestingly, there were 28 overlapping hallmark gene sets (including mTOR 1 signaling) between the hallmark gene sets associated with SLED1 and those enriched in HF (Table III). Moreover, the overlapping hallmark gene sets activated and inhibited in HF were also positively and negatively correlated with SLED1 expression, respectively (Figures 5D, 5 E). As illustrated in Figures 5D, 5E, the hallmark gene sets inhibited in HF and SLED1 were completely consistent, including E2F targets, the G2M checkpoint, and MYC targets v1.

\section{Discussion}

In the present study, 147 DEGs were identified between STEMI and CAD at admission, and 62 DEGs were screened for STEMI between admission and 6 months after MI. GO functional enrichment analyses were conducted to explore the potential functions of the two sets of DEGs. There were 50 overlapping GO terms between the two sets of DEGs, indicating that DEGs for STEMI were chiefly involved in neutrophil-related biological processes, such as neutrophil activation, neutrophil degranulation, and neutrophil-mediated immunity. GSEA revealed that most overlapping hallmark gene sets for STEMI were related to activation and/or infiltration of monocytes/macrophages. These results suggest that neutrophils and monocytes/macrophages may play vital roles in the pathogene- 
A

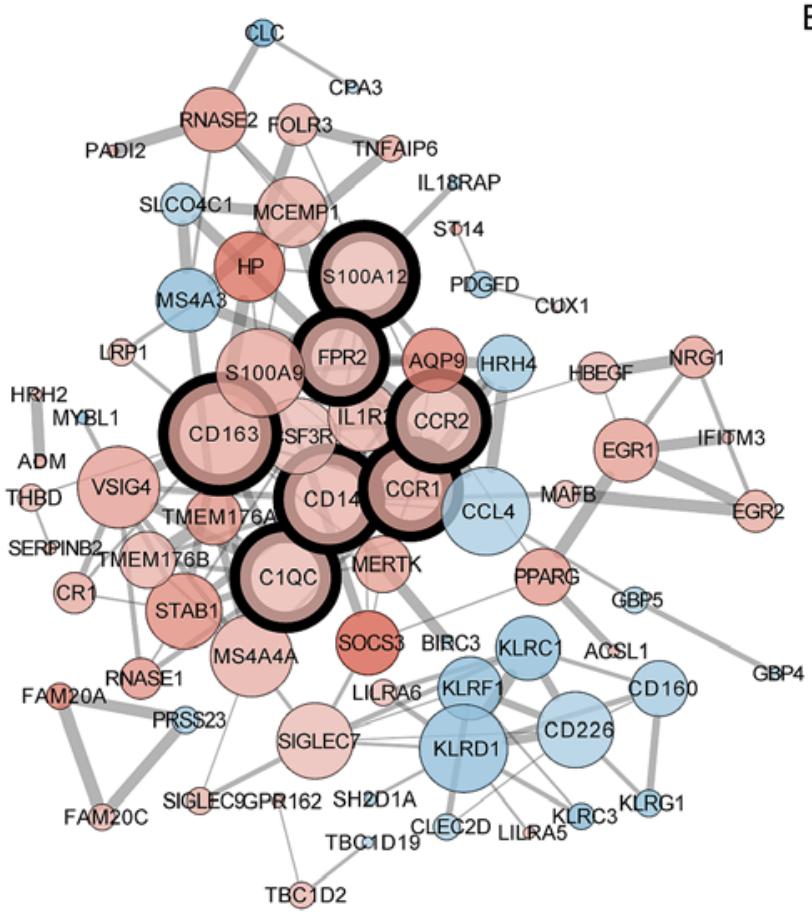

B

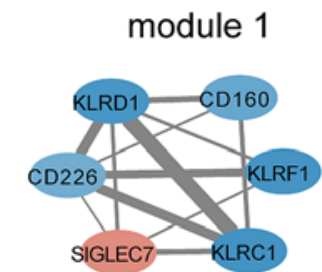

module 2
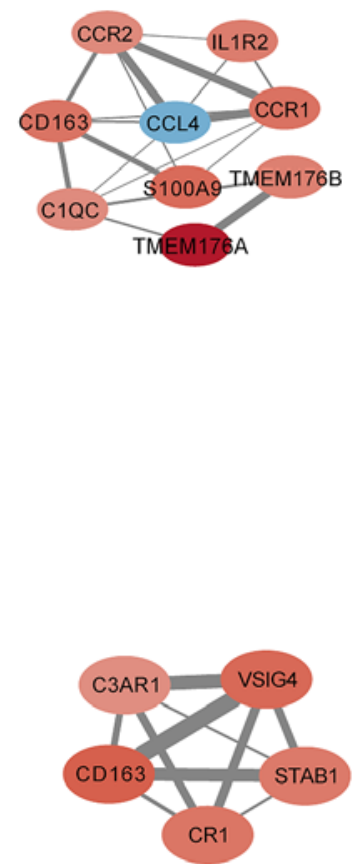

C

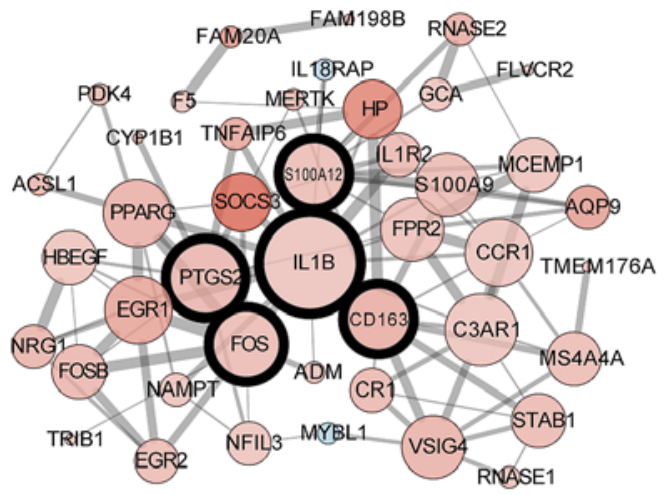

Figure 4. PPI networks construction of DEGs. A: The PPI network of DEGs between STEMI and CAD at admission was constructed using Cytoscape. B: Top two significant modules were obtained from PPI of DEGs between STEMI and CAD with scores of 4.8 and 4.5, respectively. C: The PPI network of DEGs between admission and 6 months after MI in patients with STEMI was built. D: The top significant module (score $=5.0$ ) was selected from PPI of DEGs between admission and 6 months after MI in patients with STEMI. The nodes represent the genes, whereas the lines represent interactions between genes. The gradual color change of nodes from blue to red indicates the changing process of genes from downregulation to upregulation. The width of the line denotes the combined score of the predicted interaction. The size of the nodes indicates the degree of interaction in the PPI network $(\mathbf{A}, \mathbf{B})$. Nodes with the black outline stand for the hub genes.

sis of MI. After MI, neutrophils are the first cell line to massively invade the myocardium, followed by monocytes, ${ }^{18)}$ which further differentiate into M1 or M2 macrophages. Neutrophils and the M1 subtype dominate the early inflammatory phase and secrete pro-inflammatory cytokines, causing damage and removing dead cells and debris, whereas the M2 subtype accumulates during the resolution of inflammation, promoting wound healing. ${ }^{19-21)}$ However, excessive neutrophil activation may release toxic products such as proteolytic enzymes and oxygen radicals, thus, injuring cardiomyocytes. ${ }^{22)}$ A clinical study revealed that a high neutrophil-to-lymphocyte ratio predicts large infarct sizes and poor clinical outcomes in patients with AMI who undergo PCI. ${ }^{23)}$ Animal experiments have indicated that neutrophil depletion reduces the myocardial infarct size, suggesting neutrophil-mediated injury. ${ }^{24,25)} \mathrm{Be}-$ sides, previous studies indicated that excessive activation of M1 macrophages promotes ventricular remodeling and 

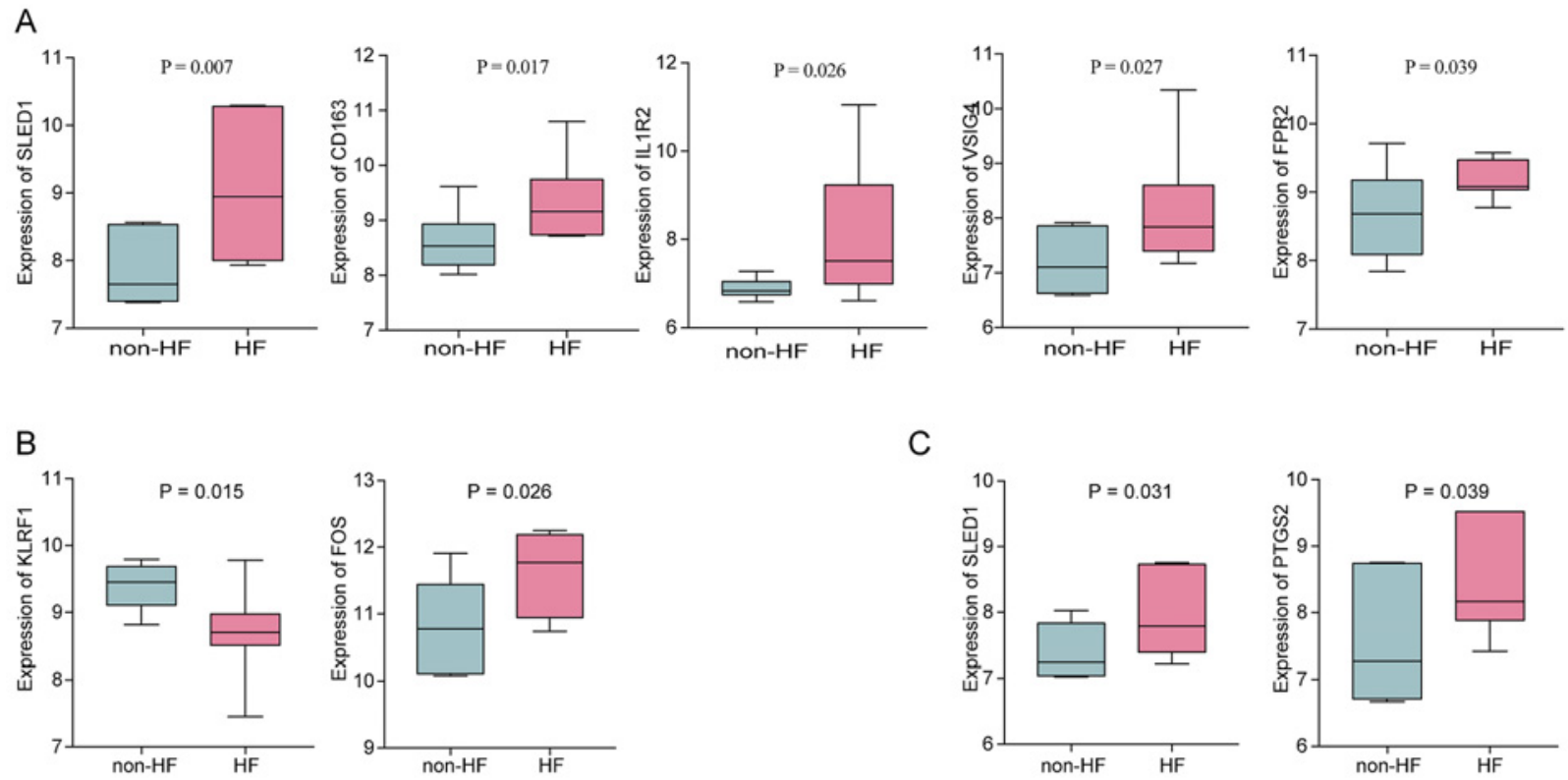

D

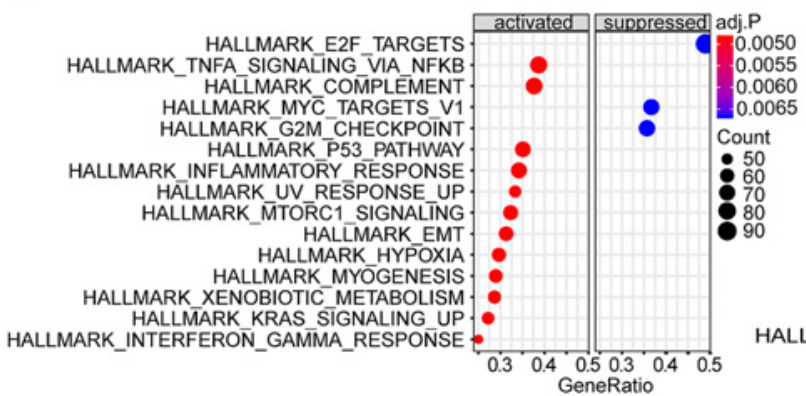

$\mathrm{E}$

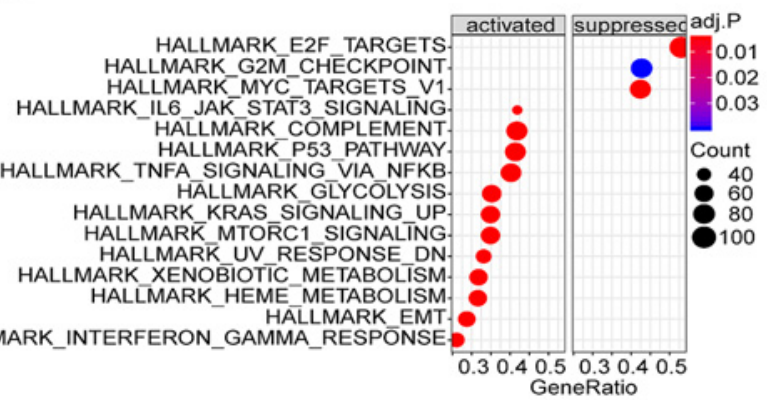

Figure 5. Biomarkers and mechanism analysis of post-infarct HF. DEGs between patients with and without HF were identified from 29 crucial genes at admission (A), discharge (B), and 1 month after MI (C). $P<0.05$ was considered statistically significant. D: GSEA of entire genes according to the $\log 2$ fold change of the gene expression ratio between patients with HF and those without HF at admission. E: GSEA of entire genes according to the correlation coefficient of SLED1 and other genes of the gene expression ratio between patients with and without HF at admission. The size and the color intensity of a circle represent the numbers of enriched genes and adj. $P$, respectively (D, E). Adj. $P<0.05$ was considered statistically significant.

HF after MI, and strategies modulating macrophage polarization have been proven to improve ejection fraction after $\mathrm{MI}{ }^{26,27)}$ suggesting a pivotal role of M1 macrophages in post-infarct $\mathrm{HF}$.

SLED1 is a pseudogene of the lncRNA class. This study demonstrated that SLED1 was upregulated in patients with post-infarct HF. Ishii and her colleagues found that SLED1 was significantly upregulated in peripheral blood cells of patients with systemic lupus erythematosus and speculated that it may be associated with systemic inflammation. ${ }^{28)}$ Also, the inflammatory response plays an important role in adverse remodeling and heart failure following MI. ${ }^{2930)}$ Therefore, we speculate that SLED1 may contribute in the progression of post-infarct HF through the inflammatory response. In the present study, we found that activation of mTORC1 is specific for post-infarct HF, and SLED1 might activate mTORC1. Interestingly, mTORC1 activity is increased in both SLE and HF, and its blockade is therapeutic. ${ }^{31-33)}$ Studies indicated that mTORC1 regulates macrophage polarization by promoting the M1 subtype and inhibiting the M2 subtype. ${ }^{34,35)}$ Disruption of mTORC1 activation in macrophages inhibits M1 macrophage-mediated inflammation. ${ }^{36}$ Considering a key role of M1 macrophages in post-infarct HF, we considered that SLED1 may promote M1 macrophage polarization and inflammation by activating mTORC1, leading to post-infarct HF. Autophagy is generally considered to be cardioprotective. mTORC1 has been shown to inhibit autophagy and promote heart failure, whereas inhibition of mTORC1 activation enhances autophagy and then mitigates HF. ${ }^{37)}$ Obviously, SLED1 may also promote heart failure after MI by regulating autophagy.

In addition, GSEA showed that genes involved in E2F targets, the G2M checkpoint, and MYC targets v1 were inhibited in post-infarct HF. All of these are involved in cell cycle regulation. For example, the E2F family 
Table III. The Overlapping Hallmark Gene Sets Involved in Heart Failure and SLED1

\begin{tabular}{|c|c|c|c|c|}
\hline \multirow{2}{*}{ Description } & \multicolumn{2}{|l|}{$\mathrm{HF}$} & \multicolumn{2}{|c|}{ SLED1 } \\
\hline & enrichmentScore & adj. $P$ & enrichmentScore & adj. $P$ \\
\hline HALLMARK_TNFA_SIGNALING_VIA_NFKB & 0.624618258 & 0.00484778 & 0.571373887 & 0.004609994 \\
\hline HALLMARK_ANGIOGENESIS & 0.591437343 & 0.00484778 & 0.538982289 & 0.008753501 \\
\hline HALLMARK_COAGULATION & 0.59135656 & 0.00484778 & 0.44302767 & 0.004609994 \\
\hline HALLMARK_IL6_JAK_STAT3_SIGNALING & 0.581241779 & 0.00484778 & 0.442838107 & 0.004609994 \\
\hline HALLMARK_INFLAMMATORY_RESPONSE & 0.571731609 & 0.00484778 & 0.449214934 & 0.004609994 \\
\hline HALLMARK_EPITHELIAL_MESENCHYMAL_TRANSITION & 0.532854975 & 0.00484778 & 0.356951265 & 0.004609994 \\
\hline HALLMARK_XENOBIOTIC_METABOLISM & 0.530068998 & 0.00484778 & 0.32658307 & 0.004609994 \\
\hline HALLMARK_REACTIVE_OXIGEN_SPECIES_PATHWAY & 0.500315914 & 0.01100352 & 0.380427613 & 0.043232458 \\
\hline HALLMARK_COMPLEMENT & 0.500106618 & 0.00484778 & 0.458650994 & 0.004609994 \\
\hline HALLMARK_CHOLESTEROL_HOMEOSTASIS & 0.450403792 & 0.01310444 & 0.343018847 & 0.034482759 \\
\hline HALLMARK_P53_PATHWAY & 0.444597839 & 0.00484778 & 0.373765243 & 0.004609994 \\
\hline HALLMARK_FATTY_ACID_METABOLISM & 0.444396361 & 0.00484778 & 0.296492338 & 0.020926339 \\
\hline HALLMARK_KRAS_SIGNALING_UP & 0.441842638 & 0.00484778 & 0.346315356 & 0.004609994 \\
\hline HALLMARK_PROTEIN_SECRETION & 0.43900199 & 0.00484778 & 0.498234018 & 0.004609994 \\
\hline HALLMARK_APOPTOSIS & 0.423140738 & 0.00484778 & 0.403838539 & 0.004609994 \\
\hline HALLMARK_UV_RESPONSE_UP & 0.418568493 & 0.00484778 & 0.400571245 & 0.004609994 \\
\hline HALLMARK_HYPOXIA & 0.413140925 & 0.00484778 & 0.393659186 & 0.004609994 \\
\hline HALLMARK_INTERFERON_GAMMA_RESPONSE & 0.413120536 & 0.00484778 & 0.353415511 & 0.004609994 \\
\hline HALLMARK_MTORC1_SIGNALING & 0.398188677 & 0.00484778 & 0.308128459 & 0.004609994 \\
\hline HALLMARK_ADIPOGENESIS & 0.386008633 & 0.00666845 & 0.318599108 & 0.004609994 \\
\hline HALLMARK_HEME_METABOLISM & 0.38273054 & 0.00666845 & 0.301756507 & 0.004609994 \\
\hline HALLMARK_GLYCOLYSIS & 0.365533466 & 0.01210287 & 0.305862863 & 0.004609994 \\
\hline HALLMARK_ESTROGEN_RESPONSE_LATE & 0.348634823 & 0.0139237 & 0.284630006 & 0.014697237 \\
\hline HALLMARK_IL2_STAT5_SIGNALING & 0.345822688 & 0.0188253 & 0.272466523 & 0.014697237 \\
\hline HALLMARK_APICAL_JUNCTION & 0.321123528 & 0.03390892 & 0.271723022 & 0.014697237 \\
\hline HALLMARK_MYC_TARGETS_V1 & -0.380791823 & 0.00666845 & -0.370638076 & 0.004609994 \\
\hline HALLMARK_G2M_CHECKPOINT & -0.43698821 & 0.00666845 & -0.260710001 & 0.039840637 \\
\hline HALLMARK_E2F_TARGETS & -0.503420367 & 0.00666845 & -0.381706116 & 0.004609994 \\
\hline
\end{tabular}

HF indicates a gene set enrichment analysis between heart failure and non-heart failure at admission, and SLED1, a gene set enrichment analysis of SLED1 in heart failure at admission.

regulates cell cycle progression through the G1/S transition, ${ }^{38)}$ and inhibition of E2F targets may lead to cell cycle arrest. The G2M checkpoint prevents damaged cells and thus prevented them from entering the mitotic $\mathrm{M}$ phase. ${ }^{39)}$ The G2M checkpoint arrest induces cells to directly enter the $\mathrm{M}$ phase without repair and poses a high risk of apoptosis. Abbate and colleagues have confirmed by autopsy that the apoptotic rate at infarct sites in patients with postinfarct HF was almost four times that in patients without $\mathrm{HF}^{40)}$ highlighting the importance of apoptosis in postinfarct HF. Collectively, these data suggest that cell cycle disorder and apoptosis may play a pathophysiological role in post-infarct HF. Surprisingly, the inhibited hallmark gene sets involved in HF and SLED1 were completely consistent, suggesting that upregulated SLED1 may also be involved in $\mathrm{HF}$ attack by interfering with cell cycle and promoting apoptosis through inhibiting E2F targets, the G2M checkpoint, and MYC targets $\mathrm{v} 1$.

These data indicate that SLED1 may be a novel biomarker for post-infarct HF. Owing to its roles in the development of post-infarct HF, SLED1 may also serve as a potential therapeutic target in this disease. However, we did not conduct experiments to validate our conclusions. Further extensive investigation and experimentation are thus warranted to confirm whether SLED1 could be a biomarker of post-infarct HF and to clarify its potential mechanisms and therapeutic value.

\section{Conclusions}

Patients with STEMI and high SLED1 expression at admission may be at a higher risk of HF and are more likely to suffer from HF during follow-up. Furthermore, upregulated SLED1 may be involved in the development of post-infarct $\mathrm{HF}$ by activating mTORC1 and inhibiting E2F targets, the G2M checkpoint, and MYC targets v1. In summary, SLED1 may be a novel biomarker of postinfarct HF and a potential therapeutic target for this disease.

\section{Disclosure}

Conflicts of interest: None.

Ethical approval: This article does not contain any studies with human participants or animals performed by any of the authors.

\section{References}

1. Nabel EG, Braunwald E. A tale of coronary artery disease and myocardial infarction. N Engl J Med 2012; 336: 54-63.

2. Abubakar II, Tillmann T, Banerjee A. Global, regional, and national age-sex specific all-cause and cause-specific mortality for 240 causes of death, 1990-2013: a systematic analysis for the 
Global Burden of Disease Study 2013. Lancet 2015; 385: 117 71.

3. Szummer K, Wallentin L, Lindhagen L, et al. Improved outcomes in patients with ST-elevation myocardial infarction during the last 20 years are related to implementation of evidencebased treatments: experiences from the SWEDEHEART registry 1995-2014. Eur Heart J 2017; 38: 3056-65.

4. Cung TT, Morel O, Cayla G, et al. Cyclosporine before PCI in Patients with Acute Myocardial Infarction. N Engl J Med 2015; 373: 1021-31.

5. Maciejak A, Kiliszek M, Michalak M, et al. Gene expression profiling reveals potential prognostic biomarkers associated with the progression of heart failure. Genome Med 2015; 7: 26.

6. Kiliszek M, Burzynska B, Michalak M, et al. Altered gene expression pattern in peripheral blood mononuclear cells in patients with acute myocardial infarction. PLoS One 2012; 7: e50054.

7. Edgar R, Domrachev M, Lash A. Gene Expression Omnibus: NCBI gene expression and hybridization array data repository. Nucleic Acids Res 2002; 30: 207-10.

8. Barrett T, Wilhite SE, Ledoux P, et al. NCBI GEO: archive for functional genomics data sets--update. Nucleic Acids Res 2013; 41: D991-5.

9. Gautier L, Cope L, Bolstad B, Irizarry R. affy--analysis of Affymetrix GeneChip data at the probe level. Bioinformatics 2004; 20: $307-15$.

10. Leek JT, Johnson WE, Parker HS, Jaffe AE, Storey JD. The sva package for removing batch effects and other unwanted variation in high-throughput experiments. Bioinformatics 2012; 28: 882-3.

11. Ritchie ME, Phipson B, Wu D, et al. limma powers differential expression analyses for RNA-sequencing and microarray studies. Nucleic Acids Res 2015; 43: e47.

12. Smyth GK. Limma: Linear models for microarray data. bioinformatics \& computational biology solutions using $R \&$ bioconductor. New York, NY: Springer; 2005.

13. Wickham H. ggplot2: Elegant Graphics for Data Analysis. New York, Springer; 2016.

14. Yu G, Wang L, Han Y, He Q. clusterProfiler: an R package for comparing biological themes among gene clusters. OMICS 2012; 16: 284-7.

15. Subramanian A, Tamayo P, Mootha V, et al. Gene set enrichment analysis: a knowledge-based approach for interpreting genome-wide expression profiles. Proc Nat Aca Sci U S A 2005; 102: 15545-50.

16. Szklarczyk D, Gable AL, Lyon D, et al. STRING v11: proteinprotein association networks with increased coverage, supporting functional discovery in genome-wide experimental datasets. Nucleic Acids Res 2019; 47: D607-13.

17. Kohl M, Wiese S, Warscheid B. Cytoscape: software for visualization and analysis of biological networks. Methods Mol Biol 2011; 696: 291-303.

18. Shinagawa H, Frantz S. Cellular immunity and cardiac remodeling after myocardial infarction: role of neutrophils, monocytes, and macrophages. Curr Heart Failure Rep 2015; 12: 247-54.

19. Jung K, Kim P, Leuschner F, et al. Endoscopic time-lapse imaging of immune cells in infarcted mouse hearts. Cir Res 2013; 112: 891-9.

20. Shiraishi M, Shintani Y, Shintani Y, et al. Alternatively activated macrophages determine repair of the infarcted adult murine heart. J Clin Invest 2016; 126: 2151-66.

21. Troidl C, Mollmann H, Nef H, et al. Classically and alternatively activated macrophages contribute to tissue remodelling after myocardial infarction. J Cell Mol Med 2009; 13: 3485-96.
22. Frangogiannis NG. The immune system and cardiac repair. Pharmacol Res 2008; 58: 88-111.

23. Hong D, Choi KH, Song YB, et al. Prognostic implications of post-percutaneous coronary intervention neutrophil-tolymphocyte ratio on infarct size and clinical outcomes in patients with acute myocardial infarction. Sci Rep 2019; 9: 9646.

24. Vinten-Johansen J. Involvement of neutrophils in the pathogenesis of lethal myocardial reperfusion injury. Cardiovasc Res 2004; 61: 481-97.

25. Granfeldt A, Jiang R, Wang NP, et al. Neutrophil inhibition contributes to cardioprotection by postconditioning. Acta Anaesthesiol Scand 2012; 56: 48-56.

26. Wang N, Liu C, Wang X, et al. Hyaluronic acid oligosaccharides improve myocardial function reconstruction and angiogenesis against myocardial infarction by regulation of macrophages. Theranostics 2019; 9: 1980-92.

27. Tokutome M, Matoba T, Nakano Y, et al. Peroxisome proliferator-activated receptor-gamma targeting nanomedicine promotes cardiac healing after acute myocardial infarction by skewing monocyte/macrophage polarization in preclinical animal models. Cardiovasc Res 2019; 115: 419-31.

28. Ishii $\mathrm{T}$, Onda $\mathrm{H}$, Tanigawa $\mathrm{A}$, et al. Isolation and expression profiling of genes upregulated in the peripheral blood cells of systemic lupus erythematosus patients. DNA Res 2005; 12: 42939.

29. Swirski F, Nahrendorf M. Leukocyte behavior in atherosclerosis, myocardial infarction, and heart failure. Science 2013; 339: 161-6.

30. Nahrendorf M, Pittet MJ, Swirski FK. Monocytes: protagonists of infarct inflammation and repair after myocardial infarction. Circulation 2010; 121: 2437-45.

31. Oaks Z, Winans T, Huang N, Banki K, Perl A. Activation of the mechanistic target of rapamycin in SLE: explosion of evidence in the last five years. Curr Rheumatol Rep 2016; 18: 73.

32. Kato H, Perl A. Mechanistic target of rapamycin complex 1 expands Th17 and IL-4+ CD4-CD8- double-negative T cells and contracts regulatory $\mathrm{T}$ cells in systemic lupus erythematosus. $\mathrm{J}$ Immunol 2014; 192: 4134-44.

33. Sciarretta S, Forte M, Frati G, Sadoshima J. New insights into the role of mTOR signaling in the cardiovascular system. Circ Res 2018; 122: 489-505.

34. Wang C, Ma J, Xu M, et al. mTORC1 signaling pathway regulates macrophages in choroidal neovascularization. Mol Immunol 2020; 121: 72-80.

35. Covarrubias AJ, Aksoylar HI, Yu J, et al. Akt-mTORC1 signaling regulates Acly to integrate metabolic input to control of macrophage activation. eLife 2016; 5: e11612.

36. Yang K, Xu C, Zhang Y, He S, Li D. Sestrin2 suppresses classically activated macrophages-mediated inflammatory response in myocardial infarction through inhibition of mTORC1 signaling. Front Immunol 2017; 8: 728.

37. Packer M. Longevity genes, cardiac ageing, and the pathogenesis of cardiomyopathy: implications for understanding the effects of current and future treatments for heart failure. Eur Heart J 2020.

38. Iaquinta PJ, Lees JA. Life and death decisions by the E2F transcription factors. Curr Opin Cell Biol 2007; 19: 649-57.

39. Yin L, Chang C, Xu C. G2/M checkpoint plays a vital role at the early stage of HCC by analysis of key pathways and genes. Oncotarget 2017; 8: 76305-17.

40. Abbate A, Biondi-Zoccai GG, Bussani R, et al. Increased myocardial apoptosis in patients with unfavorable left ventricular remodeling and early symptomatic post-infarction heart failure. J Am Coll Cardiol 2003; 41: 753-60. 